\title{
Personal Responsibility in the Age of User-Controlled Neuroprosthetics
} \author{
Goering ${ }^{1,5}$ \\ ${ }^{1}$ Department of Philosophy \\ ${ }^{2}$ School of Law \\ ${ }^{3}$ Department of Electrical Engineering \\ ${ }^{4}$ Department of Bioengineering \\ ${ }^{5}$ Center for Sensorimotor Neural Engineering \\ University of Washington, Seattle
}

Timothy Brown ${ }^{1,5}$, Patrick Moore ${ }^{2}$, Jeffrey Herron ${ }^{3,5}$, Margaret Thompson ${ }^{3,5}$, Tamara Bonaci ${ }^{3}$, Howard Chizeck ${ }^{3,4,5}$, and Sara

\begin{abstract}
Deep-brain stimulation systems are an accepted and clinically effective form of neuroprosthetic treatment for a variety of common and debilitating neurological movement disorders: Essential Tremor, Parkinson's, and others. Most current implementations of DBS are "open-loop:" they remain active continuously, whether or not the user is experiencing symptoms. Recent research suggests that it is possible to devise more advanced systems where stimulation is delivered on demand. Their research offers a proof-of-concept for a Brain-Computer Interface-triggered DBS (BCI-DBS) system capable of detecting either signs of tremor or the user's neural commands through an additional set of co-implanted sensors. The system then delivers stimulation to meet the user's needs or demands. These technologies, however, come with a set of moral problems-in particular, problems for personal responsibility. This paper investigates whether giving users moment-to-moment neural control over their DBS system is ethically responsible given that users can make bad choices and thus harm others. We also ask what responsibilities medical professionals have have to support users as they learn to adapt to neuroprosthetic use. We guide our exploration of these issues through a series of hypothetical scenarios that BCI-DBS users may face.
\end{abstract}

Keywords-Neuroethics; Implantable Systems; Deep-Brain Stimulation; Brain-Computer Interfaces; Neurological Disorders; Parkinson's Disease; Essential Tremor

\section{INTRODUCTION}

Possibilities for improving lives through computerized augmentation of the human brain abound. In the next few decades we will likely see advanced prostheses which help restore mobility to amputees, devices that reduce debilitating chronic pain, and devices capable of restoring lost or damaged sensory organs. Indeed, simple and intermediate versions of these systems already exist. Eventually we may even see technology capable of improving cognition, expanding or reshaping the sensorium, managing emotional states, or enabling data transfer between digital and biological memory. But who will determine how these devices operate in everyday use - the user, their doctor, or someone else entirely? How do we determine who is in control or who ought to be in control? How do we determine who, or what, is responsible for the user's actions?
Though the real-world precursors to this class of technology are still in their infancy, researchers are making steady progress on merging neurological and digital systems. With an installation base of over 100,000 users, so-called "brain pacemakers," or deep-brain stimulators, represent the cutting edge of our ability to integrate computer technology directly into the human brain. Deep-brain stimulation (DBS) systems are an accepted and clinically effective form of neuroprosthetic treatment for a variety of common and debilitating neurological movement disorders. Most current implementations of DBS technology, however, lack the capacity to determine whether a user is currently experiencing pathological symptoms. These "open-loop" systems remain active continuously-whether or not user is experiencing symptoms.

Recent research suggests that more advanced implementations, where stimulation is selectively delivered depending on the state of the patient of DBS, are viable. Their research offers a proof-of-concept closed-loop system capable of detecting the onset of tremor via co-implanted sensors, then delivering stimulation proportional to the symptoms' duration and severity [1]. A closed-loop device offers obvious advantages: the minimization of adverse side effects, the capability to tune implant response to symptom severity, and extended system lifespan. There is also the possibility of using co-implanted sensors to read imagined movement intentions or other similar commands; these movement intentions can be used as neural commands for a Brain Computer Inferface (BCI), and these commands could be used to trigger or modulate the level of a DBS system. A BCI-triggered DBS (BCI-DBS) system could give the user direct, immediate control over their stimulation levels through their neural commands alone.

This paper investigates whether giving users volitional control over their DBS systems is morally permissible, given that users can make bad decisions and harm others as a result. We believe that it is not only permissible, it may in fact be advantageous when compared to the alternative of making the system's operation entirely automatic. Volitional control can promote (or even extend) the user's autonomy both by allowing the user to decide how best to make tradeoffs between disorder symptoms, side-effects of stimulation, battery life, and other 
unforeseen problems. This ability may make it, perhaps, make it easier for the user to incorporate DBS into her life.

The first section of this paper serves as a technical briefing on the proposed volitionally-controlled, BCI-DBS system. We address the history of the technology, the medical conditions it is designed to treat, the criteria for patient selection, and suggests near-future developments and directions for research. The subsequent sections of the paper tell the story of Janet Parsons. She is 64 years old, lives in Seattle with her husband and caretaker Curtis, and is living with an advanced stage of Parkinson's Disease. Her disease has not been responsive to conventional therapy, making her a prime candidate for implantation of a BCI-DBS system.

Janet, however, is not a real person-she lives in Seattle of 2018, at a time when BCI-DBS systems have entered the marketplace, but are not yet widespread. Her story forms a narrative backbone through which we explore the issues and questions that are likely to arise as BCI-DBS systems are implanted in patients. Chief among those questions: Do the benefits of giving users volitional control of the implant outweigh the risks to society and to the users' self-integrity? What types of training will be required of users before they are allowed control of the implant outside of clinical settings? What are the consequences of failing to use the implant responsibly? And, finally, under what circumstances might a patient's control of the implant be revoked?

Janet's story begins with her neurologist's recommendation for implantation. It follows her through surgery, through healing and acclimatization to the presence of the implant, and out into the world as a user of the technology. We pause the narrative at points where salient questions arise, and we use these opportunities to explore while seeking technological, ethical, or legal solutions to the problems presented. The goal here is not to find concrete answers or argue in favor of strong conclusions. Rather, our goal is to stimulate imagination, debate, and to provide a point of origin for future research and exploration. We require no great leaps of technological faith; and the reader need not suspend their disbelief. We have done our best to ground our discussion in terms of what is possible today and what will likely be possible within the next few years.

\section{A TECHNICAL BRIEFING}

Neurostimulation refers to the delivery of therapeutic stimulation, in the form of electrical current, to the body's nervous system. Systems capable of delivering neurostimulation can be referred to as neuroprosthetics.[2] These neuroprosthetics can be designed to interact with any portion of the nervous system, including the human brain. Cerebral neurostimulation has been the focus of decades of clinical research, and has played a critical role in developing our understanding of the complex relationships between the physical structure of the brain and the functions of the mind and body. Neurostimulation can cause a subject to move [3], to perceive somatosensory effects [4], and can even modulate a subject's willingness to engage in high-risk behavior [5].
The most common form of neurostimulation is known as deep brain stimulation (DBS). Used to treat neurological movement disorders such as Parkinson's Disease (PD) and Essential Tremor (ET), DBS stimulates structures near the very center of the brain, such as the sub-thalamic nucleus, with the goal of mitigating the a patient's symptoms. Although current understanding of why stimulation helps patients is limited, the systems are effective enough that the devices are widely used. In addition to intracranial lead, used to deliver stimulation to the brain, DBS systems require an implanted neurostimulator (INS) unit that houses the system's batteries and control circuitry. This is generally implanted within the chest, and connected to the stimulation leads with a subcutaneous wire running up the back of the patient's neck. This allows for replacement without the need for additional neurosurgery.

The current generation of systems are relatively simple: they deliver constant stimulation at a fixed intensity, set by a clinician. Although a patient's symptoms can be intermittent, current clinical systems lack the ability to sense their environment. They cannot modulate stimulation in real time in response to the onset or conclusion of symptoms. Instead, they provide constant stimulation at a fixed rate. As such, these current systems are referred to as open-loop systems [6].

The clinician is responsible for programming the DBS system. Because the required level of stimulation varies from patient to patient, clinicians must use an exploratory, trial-anderror process of adjustment and observation to hone in on appropriate level of stimulation-one which maximizes therapeutic benefit while minimizing side effects and battery usage.[7] But the ideal amount of stimulation varies not only from patient-to-patient but also may vary from episode to episode, as most patients experience a range of symptom severity. Current generation systems must be tuned to account for the most severe symptoms a patient may experience. Because of this, current systems waste power and require more frequent replacement of the INS, on a schedule ranging from a few months to a decade [8].

Additionally, some patients report side effects caused by the stimulation. These include somatic effects such as tingling or burning [7], impaired speech [9], and even reduced inhibition or altered judgment [5]. A patient receiving constant open-loop simulation may experience these side effects at all times. Despite these shortcomings, open-loop DBS systems are approved as an effective treatment for Parkinson's Disease [10], Essential Tremor [11], and dystonia [12]. In the near future, DBS may be used to treat Tourette's syndrome, chronic pain, and depression [13]. Studies suggest that DBS may also be an effective therapy for obesity, addiction, and dementia [14].

One solution to the shortcomings of open-loop DBS is the addition of sensors and control logic to the system. [6] Modifying an open-loop system by adding sensors and control logic is known as "closing the loop." Closed-loop DBS systems read from their environment and change their parameters in real-time. In the context of DBS, a closed-loop system (CLDBS) would deliver fine-tuned stimulation, responsive to 
the duration and severity of the patient's symptoms. It would be triggered automatically by the onset of symptoms, modulate stimulation depending on symptom severity, and withdraw stimulation when symptoms cease. This would, perhaps, improve a patient's quality of life by reducing the impact and severity of side effects and the frequency of INS replacement surgeries.

For one such system, ET patients wore accelerometers on their arms; software then interpreted the raw accelerometer data to estimate the severity of the patient's tremor in real time, and adjusted DBS stimulation accordingly [15]. This process occurred without input from the patient. Another, more advanced, subset of potential closed loop neurostimulation systems would rely not on external signals such as accelerometer data. Instead, the system would directly measure the changes in neural activity that signal the onset of symptoms to determine what amount of stimulation is required at any given moment. We can call these systems bidirectional neuroprosthetics.

This form of direct measurement has already been shown to be useful in providing adaptive deep-brain stimulation to Parkinson's Disease patients. One group used sensors embedded within the leads of a DBS system to determine when a patient may need stimulation [16]. Parkinson's Disease patients who suffer from severe bradykinesia ${ }^{1}$ generate high amplitude beta-waves at one stimulation site for DBS. When these beta waves were above the average level the patient received stimulation. The patient's movement scores were improved over the open-loop case. This indicates that by applying stimulation selectively, not only was the system saving power and reducing side effects, but was also more therapeutic.

It is important to note that the closed-loop systems described so far rely on involuntary, or non-volitional tremor signatures - the user has no input in the system's stimulation state. Patients may wish, however, to make deliberate tradeoffs between symptom reduction and side effects. For example, a patient may be willing to tolerate tremor in some situations to enable clearer speech. Patients could therefore consciously choose to adjust stimulation levels in anticipation of movements that typically cause tremor, or in order to reduce uncomfortable side effects.

Many people have been trained to use brain-computer interfaces (BCI) - these interfaces allow the user to control computer systems with modulated neural data alone [17]. There are ongoing efforts to extend BCI control to DBS systems as well [1]. The user of a BCI-triggered DBS would "think" a command; the resultant change in brain activity would in turn be picked up by the device's sensors; and the system would respond with an adjustment to their neurostimulator levels. A robust enough BCI-DBS system would be fully-implantedthat is, the user would not need to use external devices or

${ }^{1}$ Bradykinesia is a form of hypokinesia (reduction in motor function) characterized by slowness of movement. Rather than being a slowness in initiation of movement (akinesia), sensors to control their stimulation levels. Further, a BCI-DBS user would have moment-to-moment over their stimulation levels - and, as a result, their brain states. In this article, we will focus on this lattermost type of implant: a DBS system equipped with sensors and software capable of responding to neural commands in real-time. We maintain that users of BCIDBS systems may face issues that make it difficult claim responsibility for their actions while using them.

\section{Why Volitional, NeURAl CONTROL?}

Janet Parsons was fifty-two years old when her tremors started. Like many things in life, the gradual onset of her Parkinsonism went unnoticed at first. Sometimes, when reaching out to answer the phone, she'd notice her arm trembling. A moment's focus halted the tremor, and all was well. An active woman, she attributed her occasional tremors to fatigue from yesterday's workout, or to the extra cup of coffee she'd had with lunch. Or, maybe, this was just one of the subtle ways her body was reminding her that she was getting older. Janet was familiar with the symptoms of Parkinson's Disease,but she attempted to rationalize her worsening symptoms away in an attempt to avoid dealing with the possibility. Her career, her hobbies, and her independence were all threatened by a shadow of a degenerative disease with no known cure.

Then came the day when she picked up the phone and couldn't hold onto the receiver. The tremor had been getting worse for a few months, and with it, the amount of focus required to control her hands had increased. She had, for the most part, been able to manage her symptoms without significant disruption to her life. But as she stared at the telephone, she knew that she had no choice but to visit her doctor. Her diagnosis came quickly. The shadow she feared was swept aside and replaced with a tangible, inescapable thing: Janet had Parkinson's Disease, and she was going to have to adapt.

Her doctor prescribed a variety of drugs-the blue Levocar and orange Tasmar tablets became a fixture of her daily life. Her symptoms abated, and in the first few years following her diagnosis, little changed in her private and professional life. But Parkinson's is a progressive disease, and after a time the drugs and the physical therapy could no longer keep her symptoms under control. Eight years after her first visit to the doctor, she entered unofficial retirement, giving control of her share of the financial advising business she had founded to her partners.

As her condition worsened, Janet did her best to stay informed of new treatment options and therapies for Parkinson's. She learned about DBS during one of her many forays into the medical literature surrounding her disease, and discussed the possibility of implantation with her neurologist. Together, they decided that DBS was not the right option for

bradykinesia describes a slowness in the execution of movement. 
Janet. In particular, Janet worried that DBS would make little difference in restoring her independence - while her tremors might be reduced, she would likely pay for that benefit with impaired speech. The thought of trading one set of symptoms for another set of side-effects didn't seem worth it, especially given the need for invasive surgery.

At her last doctor's visit, however, Janet's neurologist told her about a new type of DBS capable of minimizing side effects while maximizing therapeutic benefit. She would be able to control the DBS system with her thoughts and might, in time, learn to control the implants almost instinctively. Together with ongoing drug therapy, this BCI-DBS system offered Janet the best of both worlds - control of her symptoms when it mattered most, with minimal side effects at other times. Janet was hopeful that, for the first time in years, she might feel as if she had some control over her disease. Most enticingly, she might be able to return to work.

For the moment, let's set aside the question of whether or not the very act of giving someone the ability to directly manipulate their brain at all is ethically responsible. For now, we will focus on whether it is ethically responsible to give users this control in light of the fact that their manipulations may cause harm. Why is it desirable for Janet to have volitional control of her implant in the first place? If we assume that she was given a choice of either a BCI-DBS or CLDBS system, why would she opt for the former over the latter? Put another way, which is better for Janet: voluntary or involuntary control?

If we recall, a CLDBS system for Parkinson's can either detect the onset of symptoms, and then apply stimulation when the user needs it, or it can apply stimulation when the user gives a volitional neural command. While a non-volitional system would manage Janet's tremor and reduce the amount of time she experiences unwanted, uncomfortable side-effects, she will still experience some side-effects. And, because the device determines when she needs treatment, those side-effects could occur at inopportune times.

For example, Janet may not be able to speak well when her DBS is active [19]. Suppose Janet is giving an important presentation at work, and her DBS begins to apply stimulation halfway through her presentation. In this case, Janet would likely rather deal with tremors than speech impairment - and it would be convenient if she could just turn the device off. The non-volitional device, however, leaves Janet out of the decision loop. With the volitionally-controlled device, however, Janet would always be in the loop. The device-in the best case scenario-would use a non-volitional control scheme until the user gives a command to turn off stimulation. This would give Janet the power to decide when she wants to deal with tremors and when she wants to deal with the side-effects of neurostimulation.

In other words, a volitionally controlled device would likely give Janet a means of self-determination that would not be available otherwise. Ryan and Deci defend Selfdetermination Theory as an explanation of why it is important to respect and bolster self-determination in clinical contexts: they argue that perceived autonomy, competence, and relatedness to others are fundamental human needs [21]. That is, if persons are not able to perceive themselves as autonomous agents that complete tasks competently, they may have trouble finding the (intrinsic) motivation they need to live productively. Several studies demonstrate positive results when clinicians use techniques that both respect and support self-determinationfor example, when encouraging people with diabetes to adhere to treatment [18] or teaching people with PD-related postural instability to perform motor tasks [22].

Further, we can say devices like these, by extension, can be designed in a way that both respects and supports Janet's autonomy. There is, however, a great deal of controversy over what autonomy is and what it means to respect it in clinical contexts. Proponents of respect for decisional autonomy [20] argue that a clinician respects a patient's autonomy when they present the patient with options, provide the patient with enough information to make an informed decision, and support the patient's decision after the fact. That is, a clinician respects a patient's autonomy when she is allowed to make un-coerced, informed decisions about her own healthcare.

Several writers argue argue that we focus to much on this patient decision-making in healthcare: they argue we must also "attend to the implementation of health care choices with significant self-management implications such as healthoriented lifestyle changes." In the health-oriented lifestylechanges approach, changes happen within the context of "cultural norms and social structures and practices affect the lives and identities we regard as valuable and possible for us." That is, it not only matters what circumstances bring a person to make the health care choices they do, it also matters that they are able to incorporate those choices into the rest of their lives. And so if we take on a relational view of autonomy, we must pay attention not only to Janet's decision to have a DBS system implanted, but also to how a DBS system changes her everyday, lived experience.

It follows that a BCI-DBS device could be designed in the service of Janet's autonomy - whether we conceive of her autonomy relationally or individually. Without volitional control of her DBS system, Janet can only take note of how stimulation affects her, then cope as best as she can until the next opportunity to consult with her clinician. With volitional control, not only does she have the option of allowing her clinician to calibrate the device, she can choose to use her stimulation in a way that meets her moment-to-moment needs. That is, when Janet has the power to decide how much stimulation her system applies and when, she has the power to decide how to live with both Parkinson's and DBS (and its potential side-effects) in her particular life: in her workplace, with her family, or even by herself. Volitional control gives her the power to take ownership of her treatment in ways she would not have been able to otherwise; and we take it that may put a person like Janet be in a better position to make judgments about her moment-to-moment needs than the system's control unit. 


\section{Training TO USE A NeUROPROSTHETIC}

Time has passed since Janet's surgery. Eight weeks after being released, she visits a clinical neurologist, Dr. James Bishop. Dr. Bishop will be responsible for activating the implant, tuning it, and teaching Janet how to use the system to control her tremors. Her first visit is quite straightforward: the system is activated, and Dr. Bishop walks Janet through a series of basic motor tasks as he manually adjusts the stimulation level. Janet goes home that night with her DBS system running in open-loop mode and applying a lower level of stimulation. This acclimates Janet to the sensation of implant working, and allows her to see the system's benefits while learning to cope with limited side effects.

At her next visit, Janet is eager to begin learning how to control the system. Her hands are less shaky than they have been in years, and that was at less-than-peak stimulation! Dr. Bishop begins by adjusting several of the device's parameter settings in open-loop mode. He then switches the implant from open-loop to closed-loop mode, and walks her through a set of controlled tasks. Janet notices a difference in her symptoms as system increases stimulation in response to her tremor.

Eventually, Dr. Bishop leads Janet to a computer, places a transcutaneous communications unit over her INS module, and opens a program on the desktop. A small cube appears on the screen and begins to change colors and pulsate as it rotates. Dr. Bishop walks Janet through the previous set of exercises one more time, and Janet watches while the cube's color shifts as the sensors in her brain detect the onset of tremor, and the cube grows as the level of stimulation increases. The system is then put into volitional-control mode, and Janet practices growing and shrinking the cube-first without stimulation, and then with it.

Over the next several weeks, Janet becomes an expert at the cube game, and several others besides. She learns what she can and cannot do with the implant, how to recognize the onset of symptoms and quickly control them, and how to make decisions about whether stimulation is appropriate at a given time. Eventually, Dr. Bishop decides to leave the stimulator in BCI-trigger mode when she goes home, and Janet is free to use the implant as she sees fit.

Volitional control poses new challenges for patients because the system will require a basic level of skill to operate. Janet will need to learn to modulate her brain activity in the same way that people learn new motor or cognitive skills such as driving a car or doing math problems. There is a significant body of literature, discussed above, indicating that a person can learn to use their brain's electrical signals to control artificial devices using brain-computer interface (BCI). The coimplanted sensors of a BCI-DBS system form just such an interface, and ought to allow Janet to control her stimulation merely by thinking about it. We suggested earlier that a BCI-
DBS system could respect, support, or even extend the user's autonomy. This, however, means that Janet also has responsibilities that she did not have before. Janet, for instance, knows (or should know) ${ }^{2}$ better than to use a device that can cause harm to others without training. We could frame this responsibility as a duty to not use the BCI-DBS system until she was able to control it properly, and she ought to be held accountable - both morally and legally - for any resulting harm.

But this also means that Janet's clinicians, loved-ones, and colleagues share responsibilities with her they would not otherwise. Prior to being taught how to use the BCI-DBS system, Janet lacks the capacity to use the device responsibly. If Janet inadvertantly harmed someone because her lack of training the parties who would be responsible for making training available to her ought to take some share of the responsibility for Janet's mistake. That is, none of the parties involved with Janet's care- nor the regulatory bodies governing the implementation of neural implants, the implant's manufacturer, the medical center, Dr. Bishop, and so on-should expect Janet to be able to use the system responsibly without adequate training.

For the time being, we do not need to invoke some notion of collective responsibility [23] or shared agency [24] in order to describe how responsibility is shared between Janet, her clinicians, and her contemporaries. It is likely that, upon consideration of future cases similar to Janet's, we will find even more difficult-to-parse relationships between patient populations, neural engineers, device manufacturers, clinicians, regulative bodies, legal professionals, and the public at large. These complicated relationships are, however, outside of the scope of this paper. For the moment, it should be sufficient to identify several obligations people may have to Janet.

Their chief responsibilities at this stage are twofold. First is their responsibility to Janet, to train her to use the implant. She cannot make use of the system without training, and to deny her the ability to use the implant properly would violate her expectations of receiving a better quality of life through the device that she requested and they provided. Second, the responsibility to train Janet also extends to third parties, as without training Janet is more dangerous to others than she would be with it. Initially, the institutional actors would bear a significant responsibility to third parties - this is why training occurs in isolation. As Janet becomes more capable of controlling the implant, she also gains the knowledge and experience necessary to make decisions about her stimulation. Eventually Janet will reach a point at which she is competent enough to use the implant in her day-to-day life, outside the clinical setting. A threshold point will be reached where, absent one of the scenarios described above, it will be presumed that the institutional parties no longer bear any significant responsibility for harms caused by Janet's actions. This point may not be contemporaneous with Janet being allowed day-to-

${ }^{2}$ It is safe to presume that Janet would be informed of the risks involved in using the BCI-DBS system as part of the informed consent process prior to surgery. 
day control - there may be a period of overlap where one could claim that her training was inadequate, and, since the training was administered by the institutional parties, they are still responsible.

We should note that at this point in time Janet's responsibility to others still exists, whether or not she is in a position to fail in her responsibilities. We should expect that Janet's training regimen. The most responsible course of action would be to allow her to control the system only while in isolation (most likely at her clinician's office); the clinician would revert the system to either an open-loop setting or disable the system entirely while she is at home or in public. Only when Janet has demonstrated she can use the system competently, she would be allowed to operate it in everyday life.

There is, however, the risk that Janet's system could cause harms that she is not responsible for. First, Janet's system could malfunction because of a manufacturing defect or faulty design. If Janet's surgeon implanted a defective device in her, and if that defect caused a harm (either to Janet or someone else), it would make little sense to hold Janet responsible, so long as she was unaware of the defect. This defect may manifest as an unanticipated malfunction causing an excess or deficit in stimulation. Second is the possibility of a programming error by the clinician. The clinician is responsible for setting the device's parameters for responsiveness, and maximum/minimum stimulation levels. Incorrect parameters (say, resulting from clinician error during periodic post-training adjustments) may cause Janet to be unable to control her implant appropriately. For example, the clinician may accidentally reduce the maximum amount of stimulation Janet's implant can deliver. Later, unaware of this change, she attempts a dangerous motor task-say, pouring a cup of coffee for her husband. As she pours, she begins to tremor. She attempts to activate her implant, but it does not respond, and she burns her husband's hand. Janet has done no wrong here; she had no reason to know the implant was not functioning properly. Finally, Janet may be presented with a no-win situation - one in which her implant is simply incapable of responding appropriately. While she could be expected to identify circumstances like these through training, it is possible that Janet may find herself in a situation that exceeds the ability of the implant to respond to her commands. If this situation is entirely unforeseeable, she should not bear responsibility for the consequences (and, arguably, neither should the institutional parties).

Thorough testing and training ought to minimize these risks, and is another factor weighing in favor of conducting training in isolation. Any defects in the device would hopefully become apparent during this period. Additionally, any time a change is made to the device's programming, Janet should be required to demonstrate competence by completing training exercises. Training should focus not only on teaching Janet how to adjust her stimulator, it should also help her decide when it is appropriate to make adjustments. Training games might be designed to help Janet practice making decisions about stimulation and observing the consequences - this should allow her to develop a deeper understanding of the relationship between her stimulator use and her social context. Finally, we need to decide how proficient Janet should be before she is can use her implant without supervision, and we need to decide how best to gauge her proficiency.

\section{NegligenCE IN THE USE OF NEUROPROSTHETICS}

Janet has been living with full control over her BCI-DBS system for almost a year, and has been gradually getting back to her old life. While her PD symptoms are still a significant consideration in her day-to-day life, her implant has allowed her to engage in many activities that, prior to surgery, were impossible. Her increase in fine motor control has allowed her to type for the first time in years, and she has returned to work. Her improved mobility allows her to go hiking again. And, perhaps most importantly, she is able to drive again.

On a Saturday afternoon, Janet is driving her grandchildren, Kevin and David, to her daughter's home in Redmond. Without her implant, Janet would not be able to drive, and she has turned up her stimulation to a level that allows her full control over the vehicle. Having taken several car rides with Janet since her surgery, Kevin and David have learned that she cannot speak well while she is driving - the implant, while allowing her to drive, significantly impairs her speech. Mischievous children that they are, it did not take them long to learn that if grandma cannot speak, she cannot scold them. They've decided to take advantage of this car ride to settle a long-running dispute over who can make the more annoying noise.

Janet has had enough. She looks at the road around her, and sees nothing that presents itself as an immediate danger. Chastising the children will only take a moment, and so she reduces her stimulation so that she can speak. Her hands begin to shake, but she loosens her grip on the wheel so as not to transmit her tremor into the car's controls. As she turns towards the back seat, however, she notices a large piece of debris in her lane. She wills her implant to increase stimulation, but the system is not designed to respond instantly-ramping stimulation up or down takes several seconds. Unable to take evasive action, Janet's car hits the debris, and she swerves across several lanes of traffic as she tries to recover, sideswiping another motorist and causing both of them to hit the median divider. Janet and the children are thankfully unhurt, but the driver of the other vehicle suffers a broken leg and several other minor injuries. Janet's insurance agency denies the other driver's claim, arguing that Janet's condition voids her coverage, and that she never should have been driving in the first place.

Negligence is commonly defined as the failure to exercise reasonable care under the circumstances. This failure may take the form of an act (doing something that a reasonably careful person would not do) or an omission (a failure to do something that a reasonable person would do). This requirement is commonly known as the duty of care. We might say that a person breaches this duty when they engage in conduct that creates a foreseeable risk of harm to others. Should another 
person come to harm as a result of the breach, the negligent actor is liable for that harm. In determining whether or not someone was negligent we must consider whether, where a harm has resulted, a reasonable person would have taken a different course of action. With the matter at hand, it is clear that the user of a BCI-DBS system must exercise ordinary care to use the system so as to avoid foreseeable harms to others.

This is not as straightforward as it may seem, however, as Janet's available choices for reasonable action vary with the amount of stimulation she receives. What may be reasonable while she is receiving stimulation may very well be unreasonable when she is receiving no (or less) stimulation. Further, Janet's

Let's begin by considering the obligations of an untreated Parkinson's patient. We will, then, take stock of Janet's obligations when she uses either a non-volitional and BCItriggered DBS system. The law recognizes that physical disabilities such as Parkinson's Disease change the scope of what may or may not be reasonable for a person to do. This is not to say that a Parkinson's patient is somehow less culpable for their actions. Rather, the nature of the disease may make things that are reasonable for an able-bodied individual to do unreasonable for a PD patient to do, and vice-versa. For example, prior to surgery, it would have been unreasonable for Janet to drive at all during moderate to late stages of Parkinson's. ${ }^{3}$ Her responsibility in this circumstance is clear: driving would present a foreseeable risk of harm, and she could avoid that harm by refraining from driving. Therefore she should not drive. Her symptoms are unpredictable, and, as such, her responsibility to avoid driving would have always been in place - because she could become unfit to drive at any time, she should never operate a vehicle.

An open-loop DBS system would present the opposite circumstance. Here, Janet's tremor would be sufficiently controlled: it would not be unreasonable for her to expect that she can drive without incident. The fact that open-loop stimulation is constant and of fixed intensity suggests that this would always be the case - she could rely on the system to continually treat her symptoms, and so she could drive with the confidence that she would not have some sudden bout of tremor that could lead to an accident. As long as the implant continues to function, Janet is safe to drive.

Closing the loop, however, complicates matters. A nonvolitional closed-loop system may mitigate tremor as effectively as an open-loop system - an ideal device with an ideal algorithm would respond perfectly to symptoms. But current device designs are not ideal: there will likely be a difference between the amount of stimulation needed to treat the Janet's symptoms and the amount of stimulation the system applies. Further, the implant effectively decides for itself when stimulation is (or is not) needed, and she may find it hard to predict when the system would apply stimulation. These conditions mean, taken together, that the implant might unexpectedly alter Janet's stimulation level and leave her untreated when at inopportune times. If this happens while she is driving, it may in turn affect her ability to drive safely. We might then say Janet therefore has a duty to act as if the implant is not in place-restricting her range of possible actions in the same way that an untreated PD patient would.

Conversely, Janet would also have to avoid or develop ways to deal with situations where the unexpected presence of stimulation, rather than its absence, presents a risk of harm. DBS, in addition to enabling her to do certain things (namely those things requiring fine motor control), disables her as well; she pays for her abilities by making sacrifices in other areas of her functioning. Janet's ability to communicate effectively is perhaps the simplest of these situations to envision - she would have a duty to avoid or learn to cope with any situation in which a sudden, unexpected reduction in her ability to speak could cause harm to others. This is a restriction not incumbent upon untreated PD patients - it may be said that implantation of a non-volitional, CL-DBS system presents Janet with fewer options than she would have had she decided to forego surgery. This would be an undesirable outcome if Janet underwent DBS therapy to resume life activities that PD makes difficult otherwise. This, however, would be desirable if Janet's goal is to be relatively tremor-free while conserving battery.

A BCI-triggered system should give Janet the ability to negotiate situations that would be difficult if her stimulator were in either an open-loop or a closed-loop non-volitional mode. Janet's responsibilities seem apparent prima facie. If Janet is a skilled BCI user, she may be able to drive while using BCI-DBS - but she should avoid lowering her stimulation levels if she can. BCI-control, however, may come with its own problems. It stands to reason that as Janet gains experience, she will become more adept at modulating her stimulator's parameters. It is currently unknown how quickly a user will be able to ramp stimulation up or down, what degree of control they have in selecting a precise level of stimulation, or how much conscious effort Janet will need to control the system. Janet may, at first, find that BCI control requires deliberate, focused effort. However, with enough time and experience, it may become second nature for her. Janet may, in effect, develop reflexive control of her implant. Could Janet, then, be held responsible merely for experiencing the urge to talk to her grandchildren, if her implant detected that urge and reduced stimulation based on that urge? The law does not hold people accountable for their thoughts and urges alone, but would Janet have a duty to avoid circumstances where she might merely think in ways that would turn her implant off?

There are other unresolved issues as well. Janet's duty to control her BCI-DBS system mirrors the duty borne by any operator of a potentially dangerous device. We might say that the driver of an automobile has a positive duty to drive in ways that promote safety, for example by using lights and signals to inform other road users of their presence and intentions. The

\footnotetext{
${ }^{3}$ It is, however, difficult to determine if people in mild-to-
} moderate stages PD have the ability to drive safely. See [26]. 
same driver has negative duties as well, to refrain from driving in dangerous ways - for example, by not speeding in residential areas. But a BCI-DBS system is not just any device. It is, in effect, an integral part of the user's nervous system. Janet is not being asked to operate some external piece of hardware in a specific way. Rather she is being asked to alter (or abstain from altering) the state of her brain. While in actual fact Janet is controlling a device that in turn causes an effect on her brain (arguably, akin to taking mind-altering medication), from her point of view this process is transparent. She simply thinks a command, and her tremor changes in intensity, and she experiences proportional side-effects. While the law is unlikely to make such a fine distinction, from a philosophical perspective this raises the question of whether or not a person can be obligated to maintain a given state of mind, particularly in light of the psychosocial effects that DBS may cause in patients.

\section{DEALING WITH INTERPERSONAL CHANGE}

Janet returned to work shortly after learning how to control her BCI-DBS system. It felt good to be back at the office, earning a living, and feeling useful. The depression that she had felt since she was forced into early retirement began to lift, and Janet found herself happier than she had been in years. Her time away from her profession, however, had cost her some of her edge, and the other partners at her firm decided that it would be better for her to ease back into the work by managing some of the firm's lower-risk investment portfolios.

While at work, Janet typically set her system to provide fairly high level of stimulation. In addition to needing fine motor control to type and write, she felt social pressure to minimize the outward signs of her disease while surrounded by people who were not close friends. This use pattern was in keeping with the general purpose of her implants-to allow her to resume general life functions with minimal disruption from her Parkinson's symptoms.

Janet's performance at work was competent, but after a few years of providing decent returns for her clients, Janet's partners still hadn't allowed her to take on more responsibility. Janet resented this, and vowed to prove to them that she was just as capable as she had been before the onset of her Parkinson's. She began taking a more aggressive approach to portfolio management, engaging in risky trades in an attempt to boost performance. After a time, this behavior spilled over into her private life as well. Her friends, family, and coworkers noted a marked change in Janet's personality-where her demeanor had once been composed and understanding, she became boastful and resentful of criticism.

But these changes in Janet's personality weren't the result of some simple need to prove herself. Instead, they were the outwards manifestation of DBS' capacity to induce manic or hypomanic states in patients. Her continuous use of high levels of stimulation at work influence her to take more risks than she would otherwise, and made her less receptive to criticism. Several of her friends and co-workers are worried about the change in her personality, but Janet isn't worried at all. She says she feels free to make the most of her life.

Whereas our discussion on negligence focused on the issue of whether Janet's momentary choices to use or not use the BCI-DBS system were reasonable in some instantaneous circumstance; here, we recognize that generally reasonable use of the system may result in helpful or harmful consequences for Janet's interpersonal life. Rather than altering Janet's ability to do certain things, the psychological side-effects of DBS can alter Janet's behavior, personality, and her ability to make decisions. But can Janet be held responsible for the actions they undertake while experiencing these behavior-altering side effects? And can Janet's friends and family expect Janet to change her stimulation levels because of a personality change?

There is an ongoing debate over what personal identity consists in, and whether or not DBS systems cause significant changes to their user's identities on alternative views of identity. Glannon and Klaming both argue that psychological continuity is key in determining an individual's sense of personhood and self-identity. If a person experiences a sufficiently abrupt or severe break in the "continuity of the psychological properties that constitute the self and one's experience of persisting through time as the same person" [36], it is possible that the person will feel "out of step" with what they take to be their authentic selves. Some open-loop DBS users have reported feeling less like themselves [27] [28], and several writers argue that they feel this way precisely because stimulation can cause disruptions to their lives that makes them feel "inauthentic" [30].

Francoise Baylis argues for a "dynamic, narrative, and relational" account of personal identity, where we form our identities through a series of self-narratives [31]. These narratives are not fixed: they can change as important life events occur. Our self-narratives are not entirely within our control, either. Our friends, families, communities, and cultures at large have their own narratives for us (that we can either accept or reject). All of these parties also set limits for what we can say about ourselves. The issue for Baylis, then, is not that DBS makes a person feel less like their authentic selves by disrupting their psychological continuity or making them feel alienated. The issue is, instead, that DBS may disrupt the user's authorship over their own self-narratives. In most cases, she argues, it will be at least possible for DBS users to tell stories about who they are as DBS users.

Mackenzie and Walker go further: they insist that our worry shouldn't be that DBS challenges or threatens a person's authentic self [32]. We should, instead, ask if DBS disrupts or diminishes the user's "autonomy competence," or the series of skills they cultivate in order to "achieve an integrated but dynamic self" [33]. That is, a person is autonomous when she has cultivated the ability to understand what she values, act in the service of those values, and know when her values need adjustment. DBS in any form may make it easier or more difficult to act competently, but BCI-DBS places the user in the 
position to make decisions that would disrupt their own autonomy competence.

In one case, for example, a 62 year old Dutch man began experiencing manic episodes approximately three years after being implanted with an open-loop DBS system [34][35] .Therapy with psychiatric medication failed to control his symptoms: megalomania and impulsivity. His condition eventually degraded to the point where he was no longer able to care for himself, and he was admitted to a psychiatric hospital. Adjustment of his DBS system caused the manic symptoms to abate, but in the absence of DBS the patient's Parkinsonism was so severe that he became bedridden. The three-year period between surgery and hospitalization suggests that his condition had not come on suddenly, and that his hospitalization punctuated an even longer decline into incompetency. By the time medical professionals intervened, his decision-making faculty was so compromised that the doctors considered it unethical to allow him to make decisions about his treatment while the DBS system was active. Instead, he was only allowed to make decisions about whether to continue DBS therapy while his stimulator was switched off. Eventually, he and his doctors decided that the best choice was to reactivate his DBS and have him committed to a psychiatric facility.

If this patient's symptoms were so severe that he was adjudged to be unable to make meaningful decisions for himself (let alone about his health) while the DBS system was active, what does that say about the ability of patients with BCI-DBS systems to make sound decisions while in control of their stimulation? Had the Dutch patient had a BCI-DBS system instead of an open-loop system, would he have turned his stimulation down when he began noticing adverse psychological effects? Or would his impaired decision-making abilities have driven him to leave the stimulator on? As Mackenzie and Walker argue, "an intervention such as DBS can disrupt a person's autonomy competence to such an extent that he is unable to engage in narrative self-revision" [32].

It is uncertain whether a BCI-DBS user like Janet will be capable of easily recognizing the extent and severity of any psychological symptoms. An open-loop system would cause persistent side-effects that the patient's friends and family may be able to identify. Stimulation side-effects may only appear intermittently, when the user has activated her stimulation. They may also take longer to begin to manifest, or manifest more gradually, owing to the fact that a BCI-DBS patient will spend less total time being stimulated, and they may spend much of that time receiving low-amplitude stimulation. While this may be a net benefit in the sense that the likelihood of developing adverse psychological side effects is lessened over the open-loop case, at the same time it may make detecting those side effects more difficult.

As a further complication, individuals in manic or hypomanic states often subjectively enjoy those states, despite the fact that they can observe the negative consequences of their mania. Mania is often accompanied by feelings of grandiosity or invulnerability, and so a patient may either fail to recognize, refuse to acknowledge, or feel unable to accept the negative consequences of their stimulation. In the worst case scenario, Janet may even become "hijacked" or "addicted" to the system through a feedback loop that causes her to decide to leave the implant on at virtually all times when, in the absence of stimulation, she would be able to see the negative consequences of that decision.

Fortunately, severe psychological symptoms in DBS patients appear to be rare, and Janet, with her BCI-DBS system, could be expected to have less risk than a normal DBS patient because of her reduced exposure to high-amplitude stimulation. This suggests that her day-to-day decision-making ability has a fairly high chance of being unimpaired, and it would be unreasonable to require Janet to turn off her system prior to making major decisions. But even in the event that her judgment was impaired, would it be reasonable to ask that she deactivate her stimulator prior to making important decisions? How could we draw a boundary around the class of decisions that necessitates that stimulation be turned off prior to making a decision? And what would stop a person from changing their mind when the stimulation resumed?

It may also be possible that that there are some cases in which side effects become so severe that rather than a patient being simply unwilling to turn down stimulation, they may be unable to do so. In another case [29][37], a DBS patient with Tourette's experienced severe dissociative symptoms approximately one year after implantation. This patient used a hand-held adjustment device to control his level of stimulation, but at a certain amplitude the patient began to experience effects similar to those seen in individuals with Dissociative Identity Disorder. Above a given threshold of stimulation, the patient regressed to an anxious, child-like state, and cowered in a corner of his room. Reducing the level of stimulation alleviated the dissociative symptoms, but the patient was unable to recall any memories from the high-stimulation period. It is unclear whether Janet, if she suddenly began experiencing dissociative symptoms, would be able to turn the implant off if she wanted to. Even in the event that she were able to turn down her stimulation level, she may not remember that anything had gone wrong, and may not remember her actions during the time she was experiencing dissociative symptoms.

It may be possible to design a BCI-DBS system that also detects signs of tremor. This system could, then, determine how much stimulation would be necessary for tremor control at any given moment - this would be the user's allotted stimulation "budget." Perhaps, this would safe-guard Janet from inadvertently over- or under-stimulating herself in some contexts. This type of programming, however, may cause complications in patients who unintentionally or intentionally induce tremor signatures by constantly attempting fine motor tasks. It will be crucial to help users and their families detect psychosocial effects of both neurostimulation and the various modes of controlling neuroprosthetic devices. In particular, 
care providers, engineers, and device manufacturers should ask and keep record of about users experiences using BCI-DBSthey should pay attention in particular to how they use them around others, what peoples' reactions are, how they feel about those reactions, and so on.

\section{CONCLUSION}

In this paper, we considered several possible ways neuroprosthetics may call on their users (and their users' communities) to be responsible in a variety of contexts. In each of these contexts, we found that both non-volitional and volitional control come with their own implications for what it means to be responsible and for what responsibilities are owed to the user. Each of the issues we raised could easily be the subject of its own, more exhaustive investigation. We could also produce narratives of how DBS treatment effects personal responsibility people with other disorders: for instance, Janet's story would be entirely different if she were clinically depressed. Our goal here has been to tie these issues together into a hypothetical narrative that we can use to explore this new terrain.

As neuroprosthetics research moves forward, we must pay attention to and seek out the actual narratives and predictions of DBS users as well as their friends, families, and care providers. We will also need to engage early adopters of both nonvolitional closed-loop and volitionally-controlled BCItriggered systems. The more perspectives we gather and the more narratives we create, the more fruitful our explorations will be, and the better prepared we will be to meet the challenges neurprosthetics pose to personal responsibility.

\section{DiSCLOSURE}

This work is supported by a donation from Medtronic Inc. and by Award Number EEC-1028725 from the National Science Foundation for the Center for Sensorimotor Neural Engineering. This research was also supported by the Department of Defense (DoD) through the National Defense Science \& Engineering Graduate Fellowship (NDSEG) Program. The content is solely the responsibility of the authors and does not necessarily represent the official views of the Department of Defense, National Science Foundation, or Medtronic Inc.

\section{REFERENCES}

1. J. Herron and H. J. Chizeck. "Prototype closed-loop deep brain stimulation systems inspired by Norbert Wiener." In the Proceedings of the IEEE 2014 Conference on Norbert Wiener in the 21st Century, Boston, MA, (June 2014).

2. D. Borton, S. Micera, José del R Millán, and G. Courtine. "Personalized Neuroprosthetics." Science Translational Medicine 5, no. 210 (November 6, 2013): 210rv2-210rv2.

3. Michael SA Graziano, Charlotte SR Taylor, and Tirin Moore. "Complex movements evoked by microstimulation of precentral cortex." Neuron 34.5, 841-851 (2002).

4. Romo, Ranulfo, et al. "Somatosensory discrimination based on cortical microstimulation." Nature 392.6674, 387-390 (1998)

5. H. M. M. Smeding, A. E. Goudriaan, E. M. J. Foncke, P. R. Schuurman, J.D. Speelman, and B. Schmand. "Pathological gambling after bilateral subthalamic nucleus stimulation in Parkinson disease." Journal of Neurology, Neurosurgery \& Psychiatry 78 (5), 517-519 (2007).

6. A.O. Hebb, et al. "Creating the feedback loop: Closed-loop neurostimulation." Neurosurgery Clinics of North America 25.1, 187204 (2014).

7. Kuncel, Alexis M., et al. "Clinical response to varying the stimulus parameters in deep brain stimulation for essential tremor." Movement Disorders 21.11, 920-1928 (2006)

8. Medtronic Corp, website, "System Eligibility, Battery Longevity: Neurostimulation systems for deep brain stimulation." http://tinyurl.com/ltf8jh9 (Last accessed: March 18th, 2014).

9. Marwan I. Hariz, et al. "Multicenter study on deep brain stimulation in Parkinson's disease: an independent assessment of reported adverse events at 4 years." Movement Disorders 23.3, 416-421 (2008).

10. Deuschl, Günther, et al. "A randomized trial of deep-brain stimulation for Parkinson's disease." New England Journal of Medicine 355.9, 896-908 (2006).

11. Olof Sydow, et al. "Multicentre European study of thalamic stimulation in essential tremor: a six year follow up." Journal of Neurology, Neurosurgery \& Psychiatry 74.10, 1387-1391 (2003).

12. Marie Vidailhet, et al. "Bilateral deep-brain stimulation of the globus pallidus in primary generalized dystonia." New England Journal of Medicine 352.5, 459-467 (2005).

13. Joel S. Perlmutter and Jonathan W. Mink. "Deep brain stimulation." Annual Review of Neuroscience 29, 229-257.(2006)

14. Svjetlana Miocinovic, et al. "History, applications, and mechanisms of deep brain stimulation." JAMA Meurology 70.2, 163-171 (2013).

15. Takamitsu Yamamoto, et al. "On-demand control system for deep brain stimulation for treatment of intention tremor." Neuromodulation: Technology at the Neural Interface 16.3, 230-235 (2013)

16. Simon Little, et al. "Adaptive deep brain stimulation in advanced Parkinson disease." Annals of Neurology 74.3, 449-457 (2013)

17. Eleanor A Curran and Maria J Stokes. "Learning to control brain activity: A review of the production and control of EEG components for driving brain-computer interface (BCI) systems." Brain and Cognition 51, 326$336(2003)$

18. A. F. Williams, E. Manias, and R. Walker, "The role of irrational thought in medicine adherence: people with diabetic kidney disease," Journal of advanced nursing, 2009.

19. Sabine Skodda, "Effect of deep brain stimulation on speech performance in Parkinson's Disease," Parkinson's Disease, vol. (2012).

20. Tom L. Beauchamp, and James F. Childress. 2009. Principles of Biomedical Ethics. New York: Oxford University Press.

21. R. M. Ryan and E. L. Deci, "Self-determination theory and the facilitation of intrinsic motivation, social development, and well-being.," American Psychologist, 2000.

22. S. Chiviacowsky, G. Wulf, R. Lewthwaite, and T. Campos, "Motor learning benefits of self-controlled practice in persons with Parkinson's disease," Gait \& Posture, vol. 35, no. 4, pp. 601-605, Apr. 2012.

23. Pauline Allen, "Accountability for clinical governance: developing collective responsibility for quality in primary care." BMJ 321.7261608 $611(2000)$

24. Margaret Gilbert. Sociality and Responsibility. Lanham, Md.: Rowman \& Littlefield (2000)

25. Restatement (Third) of Torts: Phys. \& Emot. Harm (2010).

26. V. M. Heikkilä, J. Turkka, J. Korpelainen, T. Kallanranta, and H. Summala, "Decreased driving ability in people with Parkinson's disease," J Neurol Neurosurg Psychiatry, vol. 64, no. 3, pp. 325-330, Mar. 1998.

27. M. Schüpbach, M. Gargiulo, M. L. Welter, and L. Mallet, "Neurosurgery in Parkinson disease A distressed mind in a repaired body?," Neurology, vol. 66, pp. 1811-1816, 2006.

28. Y. Agid, M. Schüpbach, M. Gargiulo, and L. Mallet, "Neurosurgery in Parkinson's disease: the doctor is happy, the patient less so?," Journal of Neural Transmission, vol. 70 (Supplemental), 2006.

29. L. Klaming, P. Haselager, "Did My Brain Implant Make Me Do It? Questions Raised by DBS Regarding Psychological Continuity, 
Responsibility for Action and Mental Competence" Neuroethics 6, 527539 (2012)

30. F. Kraemer, "Me, Myself and My Brain Implant: Deep Brain Stimulation Raises Questions of Personal Authenticity and Alienation," Neuroethics, vol. 6, no. 3, pp. 483-497, May 2011.

31. F. Baylis, "II Am Who I Am': On the Perceived Threats to Personal Identity from Deep Brain Stimulation," Neuroethics, vol. 6, no. 3, pp. 513-526, Sep. 2011.

32. C. Mackenzie and M. Walker, "Neurotechnologies, Personal Identity, and the Ethics of Authenticity," in Handbook of Neuroethics, no. 10, Dordrecht: Springer Netherlands, 2014, pp. 373-392.

33. C. Mackenzie and N. Stoljar, Relational Autonomy. Oxford: Oxford University Press, 2000.

34. A. Leentjens, V. Visser-Vandewalle, Y Temel, et al. Manipuleerbare wilsbekwaamheid: een ethisch probleem bij elektrostimulatie van de nucleus subthalamicus voor ernstige ziekte van Parkinson. Nederlandse Tijdschrift voor Geneeskunde 2004;148:1394-8.

35. F. Kraemer, "Authenticity or autonomy? When deep brain stimulation causes a dilemma," Journal of Medical Ethics, vol. 39, no. 12, pp. 757760, Nov. 2013.

36. W. Glannon, "Stimulating brains, altering minds.," Journal of Medical Ethics, vol. 35, no. 5, pp. 289-292, May 2009.

37. I. Goethals, F. Jacobs, and C. Van der Linden, et. al., "Brain activation associated with deep brain stimulation causing dissociation in a patient with Tourette's syndrome," Journal of Trauma \& ..., 2008. 